\title{
Preface: Head and Neck Cancers
}

Head and neck cancers (HNCs) primarily include cancers in the oral cavity, pharynx, larynx, nasal cavity, and salivary glands and account for approximately $4 \%$ of all cancers diagnosed in the U.S. In 2017, it is estimated that these diseases affected 65,000 men and women. Among HNCs, oral and pharyngeal cancers (OPCs) constitute the major cancer type and account for 3\% of all cancers. OPCs are the sixth most common cancers occurring worldwide, with 45,780 new cases diagnosed per year in the U.S. alone, resulting in 8650 deaths. The most common type of OPC, oral squamous cell carcinoma (OSCC), is treated by surgical resection and adjuvant chemoradiation therapy. Despite advances in early diagnosis and treatment modalities, survival rate for OSCC has not improved during past decades, owing to pulmonary metastases and frequent locoregional cancer recurrence. In addition, $\mathrm{HNC}$ treatment imposes psychosocial challenges to patients and families after surgical resection and irradiation of orofacial structures, which often impair patient vital functions, such as mastication, speech, and oral hygiene. HNCs are associated with discrete environmental risk factors, including chronic exposure to tobacco and alcohol and infection with highrisk types of human papillomavirus (HPV), such as types 16 and 18 . HNCs are typically found in the aged population $(>50 \mathrm{yr}$ ) with life-long histories of tobacco and alcohol consumption. However, an emerging upward trend of OPCs has occurred, especially in younger patients who do not have a history of smoking or other environmental risks but have been exposed to HPV. Epidemiological studies have confirmed the recent surge of HPV and OPCs, whereas overall incidence of HNCs has declined in recent years. Therefore, $\mathrm{HNC}$ is a serious health problem in the U.S., and intense research endeavors are necessary and underway to improve early diagnostic tools, enhance cancer-prevention modalities, and develop nonsurgical targeted therapeutics to cure HNCs.
Emerging evidence has shown that tumors evade immune surveillance through up-regulation of discrete immune checkpoint molecules, among which programmed cell death protein ligand 1 (PD-L1) is most extensively characterized. PD-L1 is expressed in human cancers, including OSCCs, and transmits signals to suppress immune activation against tumors. Therefore, blocking these checkpoint molecules using specific antibodies has proven to be an effective therapeutic target against diverse arrays of cancer. Using animal models and clinical trials, surprising treatment outcomes have come to light. For these reasons, the U.S. Food and Drug Administration (FDA) has approved human monoclonal antibodies, for example, ipilimumab and pembrolizumab, for advanced cancers, including recurrent and metastatic HNCs. This so-called cancer immunotherapy represents new advances in cancer therapeutics that may lead to curative results for advanced cancers. Thus, this is a very exciting time for cancer biology, and the field is poised to make a giant leap forward to curing cancer, which may be within our reach in the near future. We thank the authors of the articles in this special issue of $\mathrm{HNC}$ for their contributions and sharing their expertise in various aspects of cancer biology, and we are grateful to Dr. Ben Bonavida for his guidance in completing this project.

Guest Editors:

No-Hee Park, DMD, PhD

Distinguished Professor of Dentistry and Medicine

School of Dentistry and David Geffen School of

Medicine

University of California, Los Angeles

Mo K. Kang, DDS, PhD

Section of Endodontics

Division of Constitutive and Regenerative Sciences

School of Dentistry

University of California, Los Angeles 